\title{
Diagnostic Accuracy Of Fecal Occult Blood Tests For Detecting Proximal Versus Distal Colorectal Neoplasia: A Systematic Review And Meta-Analysis
}

This article was published in the following Dove Press journal: Clinical Epidemiology

\author{
Ming Lu $\mathbb{D}^{1, *}$ \\ Xiaohu Luo $\mathbb{( D}^{2, *}$ \\ $\mathrm{Ni} \mathrm{Li}{ }^{\prime}{ }^{\prime}$ \\ Hongda Chen (D) \\ Min Dai (D) \\ 'National Cancer Center/National \\ Clinical Research Center for Cancer/ \\ Cancer Hospital, Chinese Academy of \\ Medical Sciences and Peking Union \\ Medical College, Beijing I0002I, People's \\ Republic of China; ${ }^{2}$ Department of \\ Toxicant Occupational Disease Testing \\ Laboratory, Xuzhou Cancer Hospital, \\ Xuzhou 221000, People's Republic of \\ China
}

*These authors contributed equally to this work

\begin{abstract}
Objective: We conducted a systematic review and meta-analysis aimed at evaluating the differences of diagnostic performance of fecal occult blood tests (FOBTs) in detecting advanced colorectal neoplasms located in the proximal versus distal colorectum.

Methods: PubMed, Embase, Cochrane Library, and Web of Science were searched for eligible articles published before August 17, 2018. Two independent reviewers conducted study assessment and data extraction. Diagnosis-related indicators of FOBT for detecting proximal and distal colorectal neoplasms were summarized, and further stratified by the type of FOBT (guaiac-based FOBT (gFOBT) and immunochemical FOBT (iFOBT)). Pooled sensitivities and specificities were calculated using a random effect model. Summary receiver operating characteristic curves were plotted and area under the curves were calculated.

Results: Overall, 31 studies meeting the inclusion criteria were included in this review. For gFOBT, we found no site-specific difference (proximally vs distally located) of pooled sensitivities observed in the colorectal cancer (CRC), advanced adenomas, and advanced neoplasms groups. As for iFOBT, pooled sensitivities for detecting CRC located in the distal colon/rectum were comparable with that in the proximal colon (proximal vs distal, $0.67,95 \%$ CI $0.62-0.72$ vs $0.72,95 \%$ CI 0.68-0.75), while higher pooled sensitivities for detecting advanced adenomas and advanced neoplasms located in the distal colon/rectum than for detecting those in the proximal colon were observed for iFOBT with the values of 0.24 (95\% CI $0.22-0.25)$ vs 0.32 (95\% CI $0.30-0.34$ ) and 0.25 (95\% CI $0.23-0.28)$ vs 0.38 (95\% CI 0.36-0.40), respectively. Summary receiver operating characteristic curve analyses showed similar patterns for both types of FOBT regarding the diagnostic accuracy for detecting colorectal neoplasms according to the anatomical sites of the colorectum.
\end{abstract}

Conclusion: iFOBT had higher sensitivity for detecting advanced adenomas and advanced neoplasia located in the distal colon/rectum than that for those in the proximal colon.

Keywords: sensitivity, colorectal cancer, screening test

\section{Introduction}

With an estimated 1,849,518 new cases and 880,792 deaths occurring in 2018, colorectal cancer $(\mathrm{CRC})$ is the third most commonly diagnosed cancer and fourth most common cause of cancer-related deaths worldwide. ${ }^{1}$ Current practices have suggested that screening for $\mathrm{CRC}$ is effective in reducing CRC mortality. ${ }^{2,3}$ The fecal occult blood test (FOBT) is a noninvasive CRC screening test that has been widely used in many screening programs and is recommended by current guidelines for CRC screening. ${ }^{4,5}$ Overall, there are two types of FOBTs which use different mechanisms to identify fecal occult blood. The traditional one is
Correspondence: Min Dai; Hongda Chen National Cancer Center/National Clinical Research Center for Cancer/Cancer Hospital, Chinese Academy of Medical Sciences and Peking Union Medical College, Beijing I0002I, People's Republic of China

Tel +86-10-8778-7394

Email daimin2002@hotmail.com;

hongda.chen@cicams.ac.cn 
the guaiac-based FOBT (gFOBT) for detecting peroxidase activity of heme. Another type of FOBT is immunochemical FOBT (iFOBT, also referred to as FIT) that uses antibodies to specifically detect human hemoglobin in stool, which make fecal immunochemical tests less likely to result in false-positive results due to the ingredients. The iFOBT has been shown to have better analytical and clinical sensitivity and higher detection ability in terms of CRC and its precursors, improved compliance, and cost-effectiveness, compared to gFOBT. ${ }^{6}$

Previous randomized controlled trials have demonstrated that FOBT-based screening could reduce the mortality associated with $\mathrm{CRC}$. $^{7,8}$ In a FOBT-based CRC screening, patients with positive FOBT results are scheduled to undergo subsequent colonoscopies for clinical diagnosis. Therefore, the diagnostic performance of FOBT would strongly affect the efficacy of screening. In a meta-analysis including 19 studies the pooled sensitivity of FOBT for CRC was relatively high at 79\% (95\% CI, 69-86\%), with corresponding pooled specificity of $94 \%{ }^{9}$ However, some studies have reported that the diagnostic performance of FOBT varied according to the anatomical site of the colorectum. ${ }^{10,11}$ Even with screening colonoscopy, which is considered as the gold standard for CRC screening, a much higher protective effect for distal colon/rectum than proximal colon was observed for colonoscopy-based screening. ${ }^{12}$ Therefore, the gradient in the efficacy of population-based screening according to the anatomic site of the colorectal lesions may be larger considering the site-specific sensitivities for colorectal neoplasms. Two systematic reviews in this area were published several years ago although the focus on this issue was constricted due to their limited sample size and incomplete study scope of colorectal neoplasia. ${ }^{10,11}$ Based on these studies, we conducted an updated systematic review including all relevant studies to further evaluate the diagnostic accuracy of FOBT for CRC and advanced adenomas according to different anatomical sites.

We aimed to evaluate the differences of diagnostic performance of FOBT in detecting colorectal neoplasms located in the proximal versus distal colorectum and to provide important references for designing effective FOBT-based CRC screening programs in the future.

\section{Methods}

We conducted a systematic review and meta-analyses following the PRISMA statement. ${ }^{13}$

\section{Data Sources And Search Process}

To identify potential studies reporting data on the diagnostic performance of FOBT for detecting colorectal neoplasms of the proximal and distal colon/rectum, two independent investigators searched PubMed, Embase, Cochrane Library, and Web of Science to retrieve studies published before August 17, 2018 using the following combined search terms: [colorectal (or) colon (or) rectum] (and) [cancer (or) neoplasm (or) carcinoma (or) adenoma (or) malignancy] (and) [faecal immunochemical test (or) fecal immunochemical testing (or) fecal immunochemical test (or) faecal immunochemical testing (or) faecal occult blood test (or) FOBT] (and) [detection (or) screening (or) detecting (or) diagnosis]. Detailed search terms and retrieval records are provided in Table S1.

\section{Study Selection}

An initial search based on the titles and abstracts was conducted to exclude studies that were not relevant to the study topic. In addition, conference abstracts without full texts or studies written in non-English language were also excluded. For potential relevant articles identified in the initial search, a full-text review was performed using the following inclusion criteria: 1) FOBT results should be reported along with colonoscopy results available as the gold standard of reference; 2) site-specific diagnosis information was provided in detail or the diagnosis-related indicators were accessible to be assessed.

\section{Quality Assessment}

Potential risks of bias and applicability of the included studies were assessed according to the Quality Assessment of Diagnostic Accuracy Studies (QUADA-2) scoring system, ${ }^{14}$ and the detailed protocol was shown in the supplementary $\underline{\text { material (Protocol 1). }}$.

\section{Data Extraction}

Assessment of the included studies was performed independently by two reviewers (ML and XL) during the whole process. When disagreement occurred, consensus was reached through discussion between the authors or referral to the third reviewer (HC and MD). The following information was extracted: year of publication, country, study setting (clinical setting: participants recruited in hospitals or clinics in an opportunistic manner; screening setting: participants recruited in communities in an organized manner), population characteristics (sample size, age, and sex, etc.), diagnostic outcomes, attributes of FOBTs (type, brand, and cut-off value), sensitivity, and specificity.

In this review, we only focused on the diagnostic accuracy of FOBT in one single round of testing. For multiple rounds of FOBT tests, only the first-round result was extracted. Sensitivity was defined as the proportion of FOBT-positive patients among those who were diagnosed with the outcome of interest. As the 
main indicator of diagnostic performance, sensitivity was extracted directly according to the anatomical site (proximal and distal colon/rectum) if reported or was calculated based on reported information. Specificity referred to the number of participants with negative FOBT results divided by the number of participants without neoplasms. For quantitative FOBT results with more than one cut-off value reported in the study, the cut-off values recommended by the manufacturer were used. Only patients with targeted tumors located in an isolated side were considered. In this review article, the proximal colon was defined as colon involving the area proximal to the splenic flexure while distal colon including distal to the splenic flexure. Advanced adenoma was defined by at least one adenoma with any of the following features: $\geq 1 \mathrm{~cm}$ in size, tubulovillous or villous components, and high-grade dysplasia. Advanced neoplasms included CRC and advanced adenomas.

\section{Data Synthesis And Statistical Analysis}

We first summarized the basic characteristics of all the included studies. Diagnosis-related indicators including sensitivity (true positive rate) and specificity (true negative rate), stratified by anatomical location were extracted or calculated and respective 95\% CIs were also reported (the Wilson method was used if not reported in the studies). We applied the random effect model to estimate the pooled sensitivity and specificity. Statistical heterogeneity was assessed by $I^{2}$ statistics. Forest plots for the pooled sensitivities of FOBT for detecting colorectal neoplasms in the proximal and distal colon/rectum were generated. Summary receiver operating characteristic (SROC) curves were plotted to assess the accuracy of FOBT for detecting proximal and distal colorectal neoplasms. $Z$ test was applied to examine the statistical difference of the areas under SROC curves according to the anatomical sites.

Subgroup analyses were also performed to estimate the effect of study setting (clinical setting vs screening setting) and type of iFOBT (qualitative vs quantitative) on the diagnostic performance of FOBT. Potential publication bias was evaluated for studies using gFOBT and iFOBT separately by Deeks' funnel plot.

All the analyses were conducted using Meta-Disc software 1.4 and Stata 12.0. Statistical tests presented were two-sided, and $P$-value $<0.05$ was considered statistically significant.

\section{Results}

\section{Literature Search Result}

As shown in Figure 1, 9066 studies were identified from the initial literature search. After removal of 3694 duplicates, a first round of title and abstract review was conducted, and 4888 articles unrelated to the review topic, 421 non-English language articles and 11 articles having no full-text were excluded. For the 52 remaining articles, a full-text review was conducted and 21 of them were excluded due to the following reasons: 1) sitespecific diagnostic indicators could not be calculated $(n=16) ; 2)$ colonoscopy conducted for participants with positive FOBT results only $(n=4)$; and 3$)$ repeated information contained in the previous articles by the same study group $(n=1)$. Finally, 31 eligible studies were included in this review.

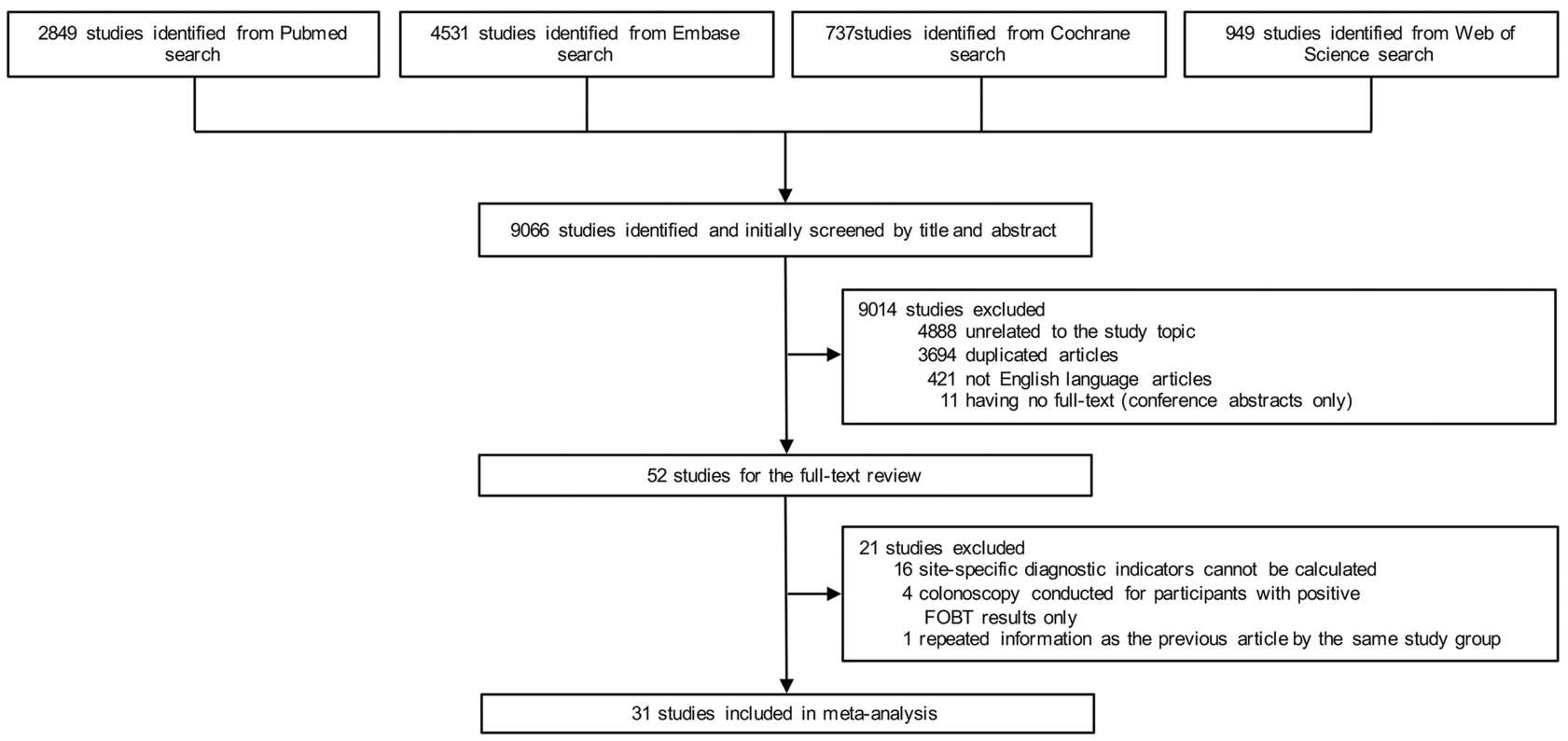

Figure I Flow chart of search strategy and selection of reports (search until August 20, 20I8). 


\section{Study Characteristics}

Table 1 shows the main characteristics of the studies included in this review. ${ }^{15-45}$ Overall, most studies were carried out in Western countries (16 of 31), while only some of them were conducted in East Asia (12 of 31). With regards to the study setting, most of the included studies (20 of 31) were conducted in a screening setting. The sample sizes varied greatly across the studies, ranging from 112 to 21,805 . The mean ages of the population in these studies ranged from 48 to 69 years. Regarding the types of FOBT, 2 studies used gFOBT, 21 studies used iFOBT, and 8 studies had results available for both types of FOBT. The brands of the FOBT also varied greatly, with a total number of 24. Among these 31 studies, 24 (77.4\%) studies had already excluded patients with incomplete bowel examination by colonoscopy, ${ }^{17,18,20-29,31-33,35,36,39,41-44}$ while 7 (22.6\%) studies did not report such exclusion. 19,20,30,34,37,38,40

\section{Diagnostic Accuracy Of gFOBT}

The summaries of the diagnostic performance of FOBT for detecting colorectal neoplasms according to the anatomical locations of all the included studies were shown in detail in Tables S2-S4. Forest plots showing the pooled sensitivities of FOBTs for detecting proximal and distal colorectal neoplasms are shown in Figures 2 and 3. For studies using gFOBT, the pooled sensitivity for detecting CRC located in the proximal colon was comparable with the pooled sensitivity of that located in the distal colon/rectum, with values of 0.60 (95\% CI 0.45-0.74) and 0.70 (95\% CI 0.57-0.80), respectively. The diagnostic accuracy of gFOBT for detecting advanced adenomas was typically low, and there was no difference between detection of advanced adenomas located at the proximal colon $(0.10,95 \%$ CI $0.07-0.14)$ and that at the distal colon/rectum $(0.11,95 \%$ CI $0.07-0.15)$ and for detection of proximally and distally located advanced neoplasms $(0.18,95 \%$ CI $0.14-0.22$ vs 0.2 , 95\% CI $0.17-0.24)$. The corresponding pooled specificities of gFOBT for CRC, advanced adenomas, and advanced neoplasms according to the anatomic site were equal, with the values of $0.88(95 \%$ CI 0.87-0.89), 0.91 (95\% CI 0.90-0.92), and 0.95 (95\% CI 0.95-0.96), respectively.

\section{Diagnostic Accuracy Of iFOBT}

For studies using iFOBT, the sensitivities for detecting colorectal neoplasms were higher than that with traditional gFOBT. For detecting CRC, comparable sensitivities of iFOBT for detecting CRC located in the proximal colon and distal colon/rectum were observed $(0.67,95 \%$ CI $0.62-$
0.72 and $0.72,95 \%$ CI $0.68-0.75$, respectively). However, higher sensitivities were observed for detecting advanced adenomas or advanced neoplasms located in the distal colon/rectum than that for the ones located in the proximal colon, with the values of 0.24 (95\% CI $0.22-0.25)$ and 0.32 (95\% CI $0.30-0.34)$ for proximally and distally located advanced adenomas, respectively, and with values of 0.25 (95\% CI 0.23-0.28) for proximally located advanced neoplasms vs $0.38(95 \%$ CI $0.36-0.40)$ for distally located advanced neoplasms. The corresponding pooled specificities of iFOBT for CRC, advanced adenomas, and advanced neoplasms were equal in terms of anatomic site, with the values of 0.95 (95\% CI $0.94-0.95), 0.93$ (95\% CI 0.93-0.93), and 0.94 (95\% CI 0.94-0.94), respectively.

\section{Summary Operating Characteristics Analyses}

We further constructed SROC curves to compare the overall diagnostic performance of gFOBT and iFOBT for detecting colorectal neoplasms located in the proximal or distal colorectum (Figure 4). For gFOBT, the areas under the SROC curve for CRC, advanced adenomas, and advanced neoplasms located in the distal colon/rectum were not significantly higher than that for those in the proximal colon/rectum (CRC, proximal vs distal, 0.853 vs 0.896; advanced adenomas, proximal vs distal, 0.588 vs 0.531 ; advanced neoplasms, proximal vs distal, 0.683 vs 0.728 ). For iFOBT, the results confirmed a significantly better diagnosis performance for advanced neoplasms (proximal vs distal, 0.760 vs $0.822 ; P=0.02$ ) located in the distal colon/rectum than for that in the proximal colon, while similar results were not observed for $\mathrm{CRC}$ and advanced adenomas (CRC, proximal vs distal, 0.929 vs 0.942 ; advanced adenomas, proximal vs distal, 0.671 vs 0.733 ).

\section{Subgroup Analyses}

To evaluate the potential effect of two factors including the type of iFOBT (qualitative or quantitative) and the study setting (clinical setting or screening setting), we further conducted subgroup analyses stratified by these factors and the results are shown in Figures $\mathrm{S} 1 \mathrm{~A}-\mathrm{S} 3 \mathrm{~B}$. Regarding the types of iFOBT, both qualitative and quantitative iFOBTs had higher sensitivities for detecting colorectal neoplasms located in the distal colon/rectum than for those located in the proximal colon/rectum. Regarding the study setting, higher sensitivities for $\mathrm{CRC}$ in the distal colon/rectum than 


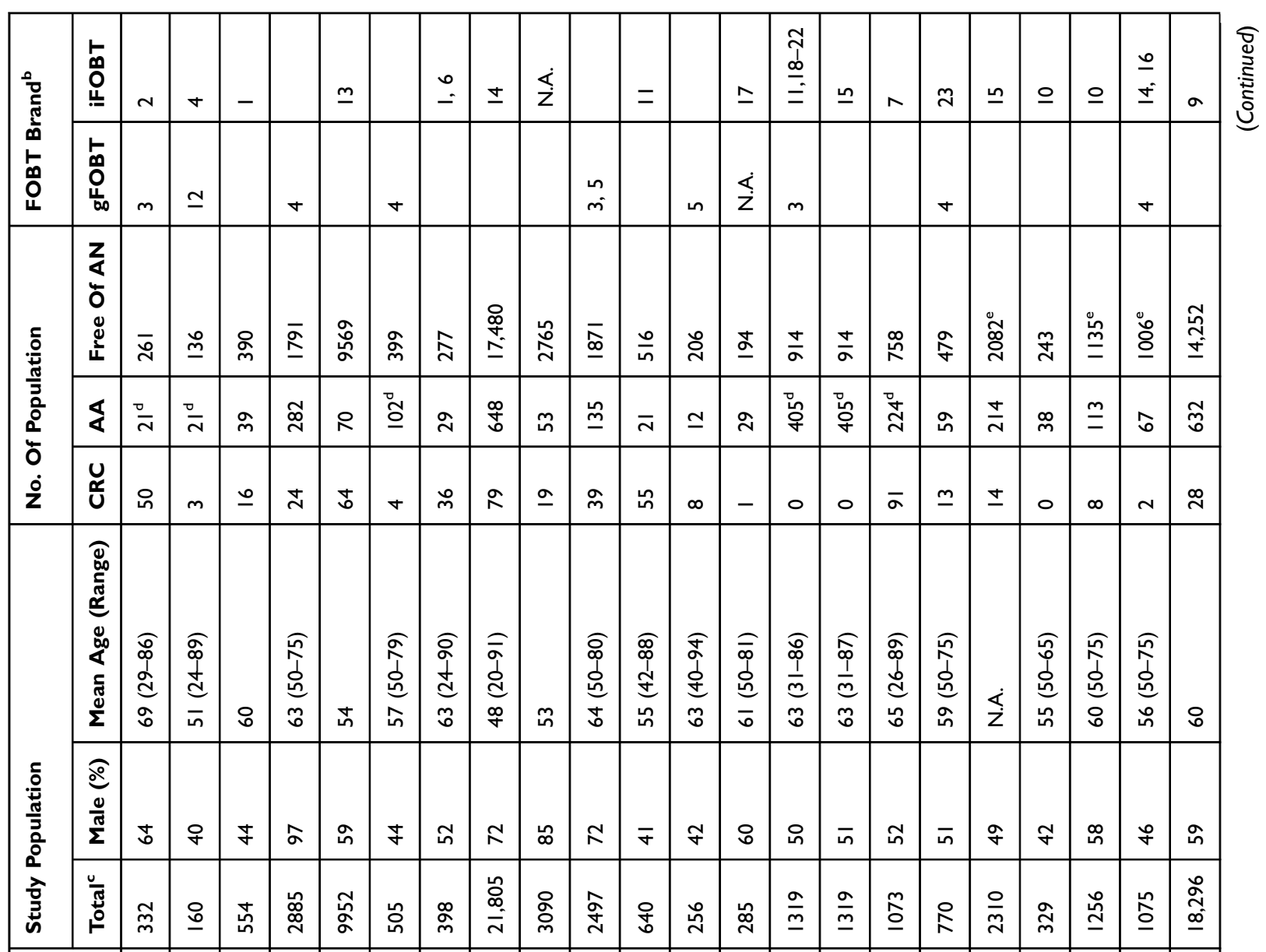

\section{㝘虽}

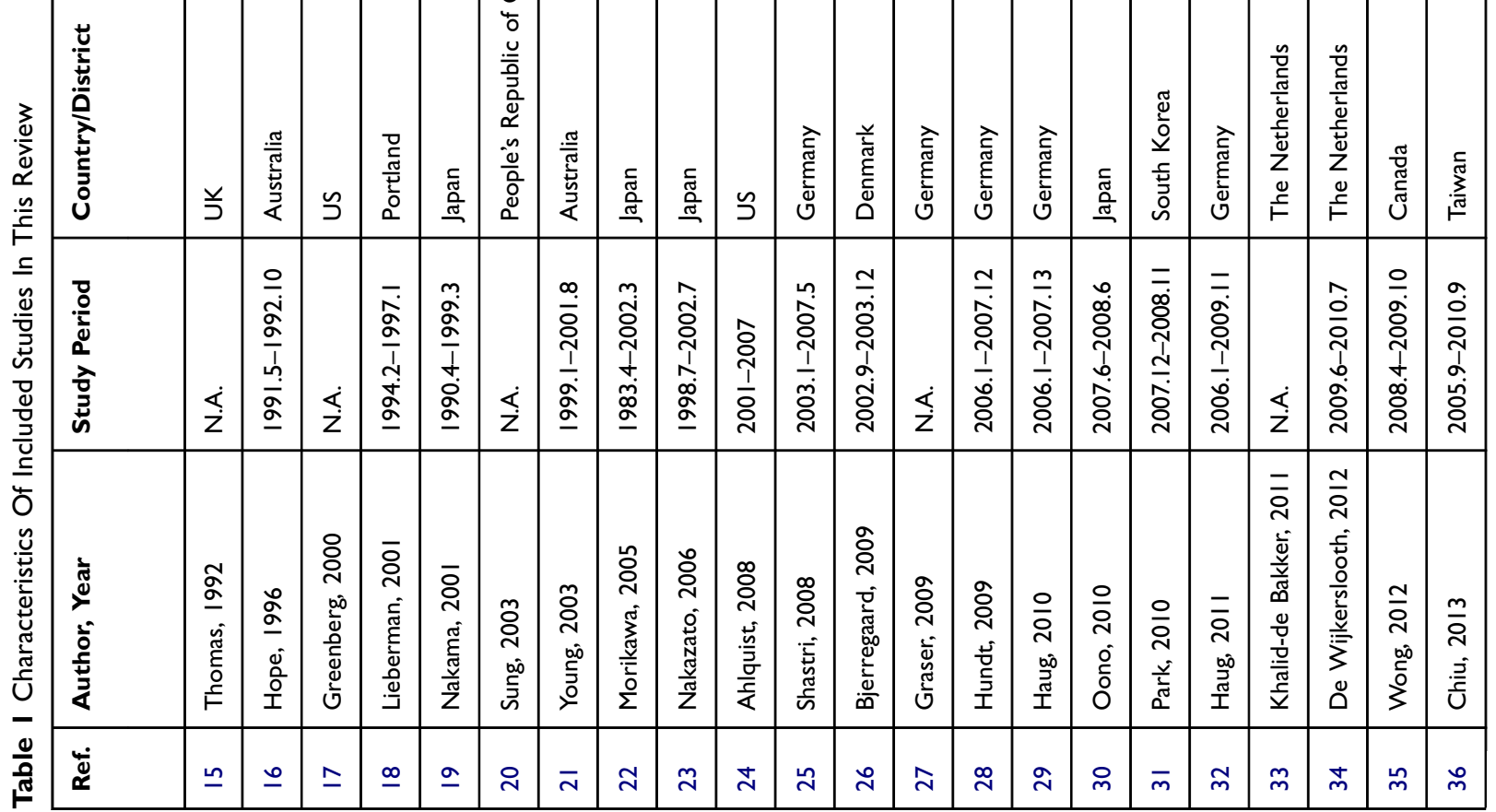




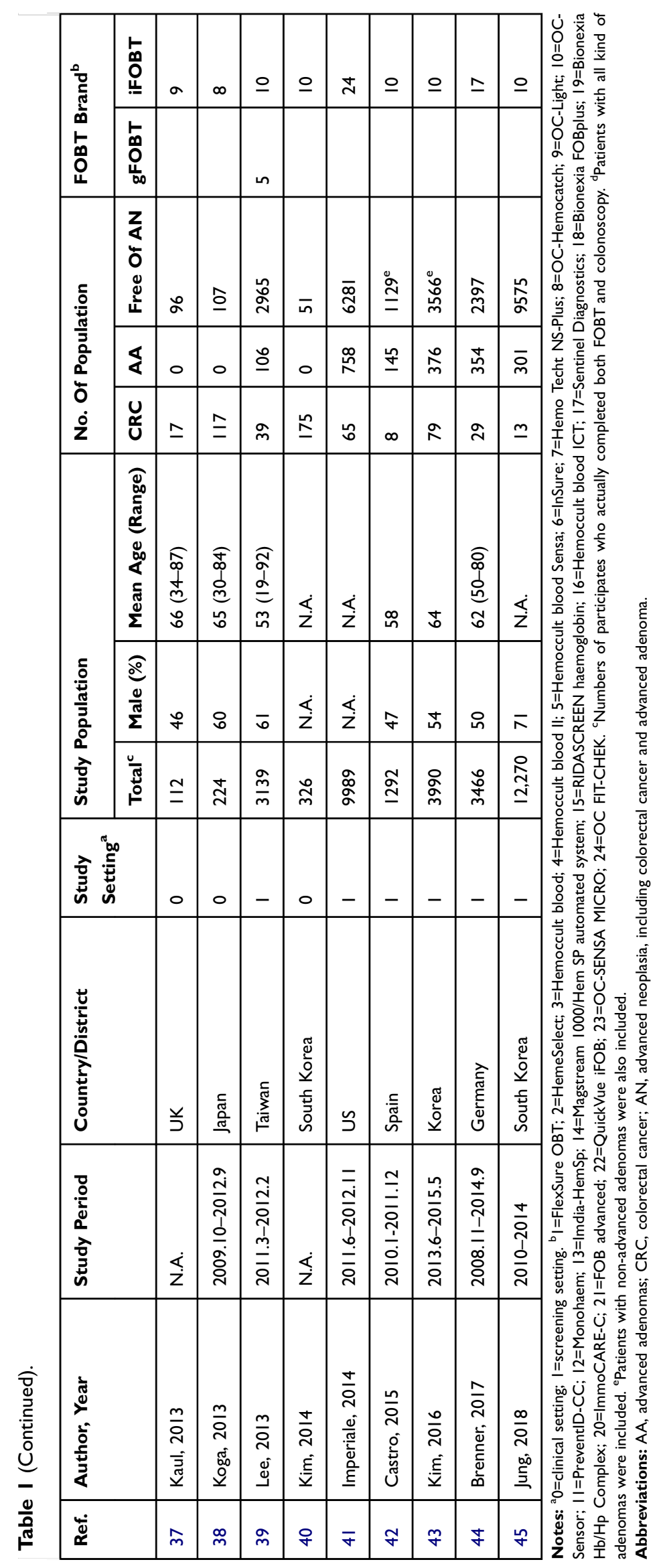




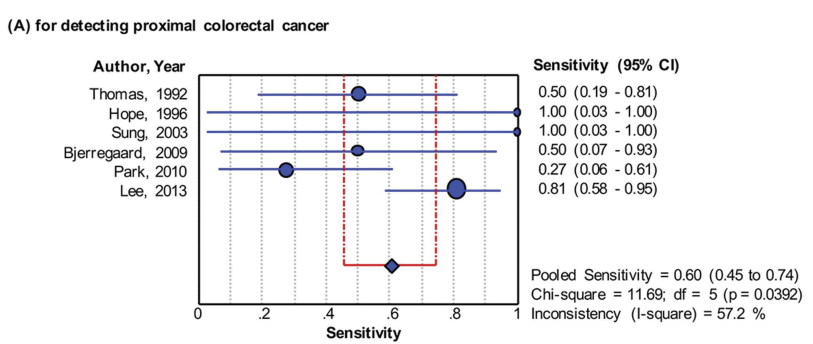

(C) for detecting proximal advanced adenoma

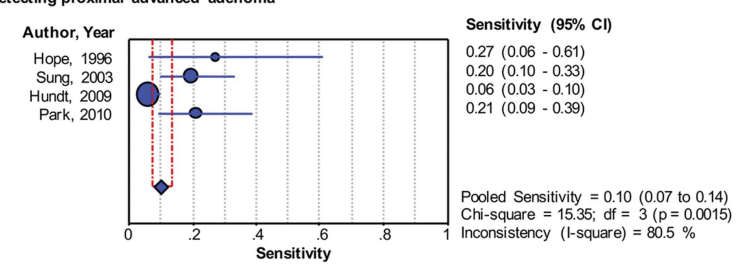

(E) for detecting proximal advanced neoplasia

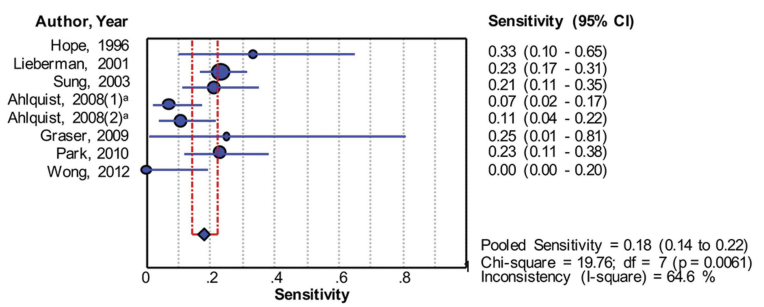

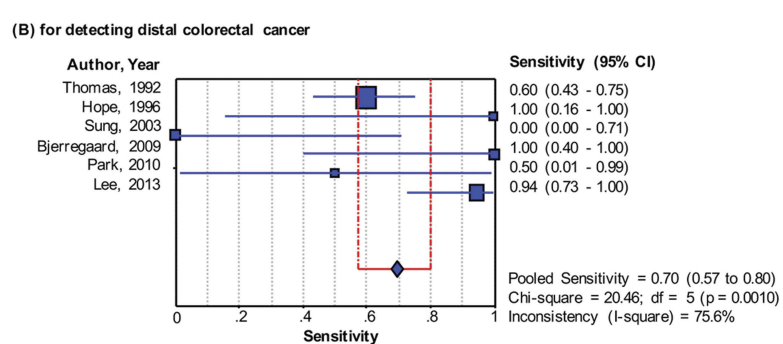
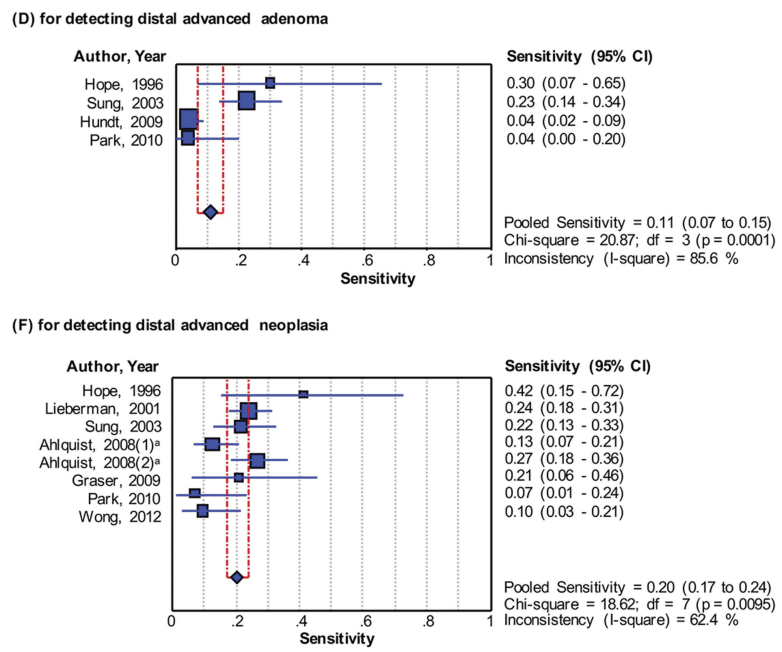

Figure 2 Forest plots for the pooled sensitivities of guaiac-fecal occult blood test on the detection of colorectal neoplasms, (A) for detecting proximal colorectal cancer; (B) for detecting distal colorectal cancer; (C) for detecting proximal advanced adenoma; d) for detecting distal advanced adenoma; (E) for detecting proximal advanced neoplasia; (F) for detecting distal advanced neoplasia.

Note: ${ }^{\text {a }}$ Ordinal numbers were applied to mark the studies which contain variable FOBT brands.

for that in the proximal colon/rectum were observed, in both clinical and screening setting subgroups, and similar results were observed for advanced neoplasms. As for advanced adenomas, higher sensitivities for distal advanced adenomas were only observed in the screening setting because the sample size was too limited in the clinical setting to calculate the corresponding pooled sensitivities.

\section{Publication Bias And Quality Assessment}

Publication bias was assessed by Deeks' funnel plots (Figure S4) and we found no strong evidence for publication bias (Egger regression tests with all $p$-values $>0.05$ ). The risk of bias assessment for included studies (Figure S5) suggested the greatest risk of bias occurred in the "flow and timing". This is mainly because 5 studies did not use identical reference standard, ${ }^{15,26,33,37,42}$ while 3 studies failed to include the whole participant group. ${ }^{9,21,40}$ The greatest concern of applicability came from the "patient selection" category, where 12 studies potentially included patients who had a history of CRC, inflammatory bowel disease and were actively bleeding, or who had a history of colonoscopy in the preceding 5 years. ${ }^{15-17,19,21,25,30,31,34,37,38,40}$ A summary of risk of bias and applicability concerns for each study was shown in Figure S6.

\section{Discussion}

In this article, we systematically evaluated the site-specific diagnostic performance of both gFOBT and iFOBT with a single test for detecting colorectal neoplasms. For gFOBT, the diagnostic accuracy of detecting colorectal lesions located in the two different colon sites were similar, with comparable pooled sensitivities and areas under the SROC curves. For iFOBT, we found that the diagnostic performance of iFOBT for detecting advanced adenomas and advanced neoplasia varied according to the anatomical sites of the colorectum, with better sensitivities for the detection of these lesions in the distal colon/rectum than in the proximal colon. As the evidences have shown that $\mathrm{iFOBT}$ is superior to gFOBT for CRC screening, iFOBT has been one of the most widely used non-invasive tests for CRC screening. However, the different diagnostic accuracy 


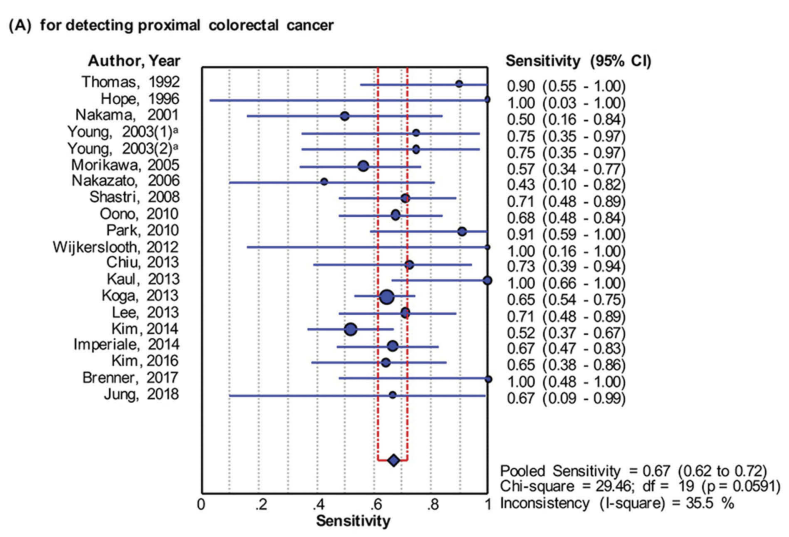

(C) for detecting proximal advanced adenoma

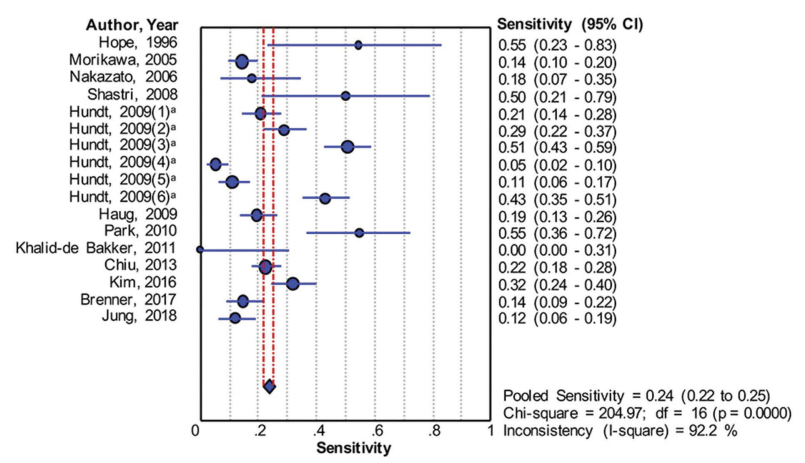

(E) for detecting proximal advanced neoplasia

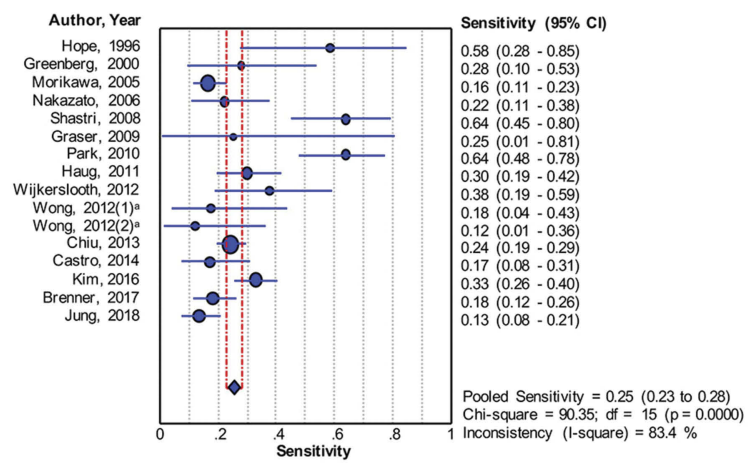

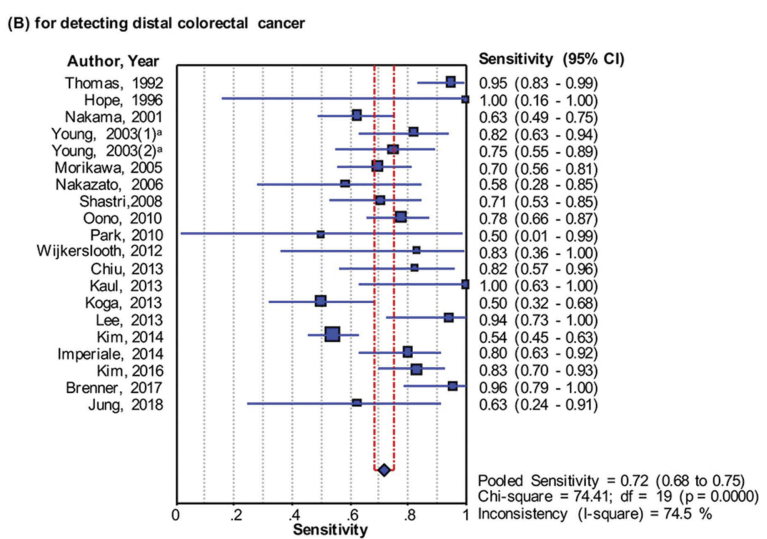

(D) for detecting distal advanced adenoma

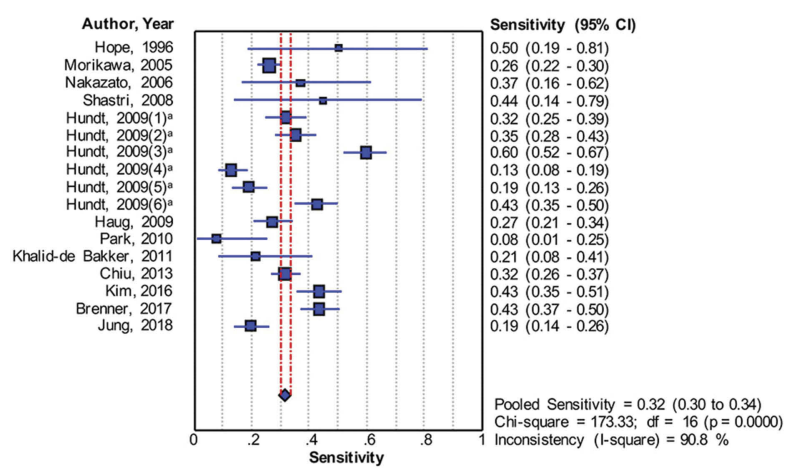

(F) for detecting distal advanced neoplasia

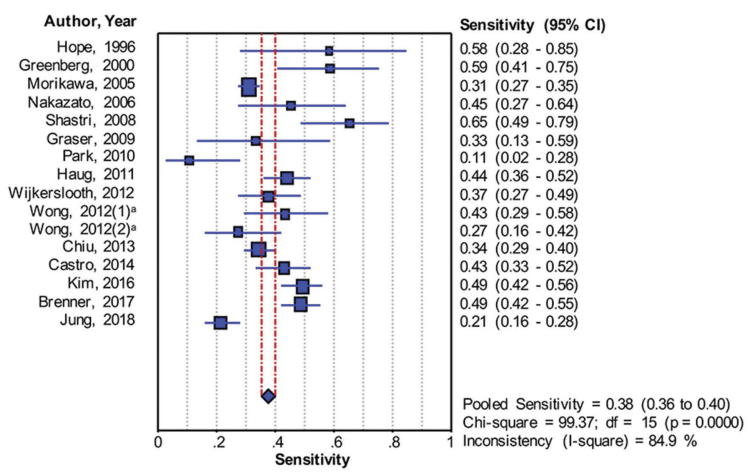

Figure 3 Forest plots for the pooled sensitivities of immunochemical fecal occult blood test on the detection of colorectal neoplasms, (A) for detecting proximal colorectal cancer; (B) for detecting distal colorectal cancer; (C) for detecting proximal advanced adenoma; (D) for detecting distal advanced adenoma; (E) for detecting proximal advanced neoplasia; (F) for detecting distal advanced neoplasia.

Note: ${ }^{a}$ Ordinal numbers were applied to mark the studies which contain variable FOBT brands.

for advanced neoplasms in terms of anatomic locations may strongly affect the efficacy of CRC screening, and the effect of this technique on long-term reduction of incidence and mortality would be expected to be stronger for distal than for proximal $\mathrm{CRC}$, which should be given attention in population-based CRC screening programs.

Our results were not completely consistent with that of the two published systematic reviews. A systematic literature review performed by Haug et al suggested a lower sensitivity of FOBT for advanced neoplasia in the right versus left colon. ${ }^{10}$ However, the results may be prone to be bias due to a lack of rigid statistical analyses. Moreover, the conclusion from a meta-analysis by Hirai et al mentioned a worse diagnostic performance of FOBT for CRC in the proximal bowel than in the distal bowel, ${ }^{11}$ but this may not be convincing because of the largely overlapping confidence intervals of the site-specific sensitivities. For our study, we conducted an updated systematic review with quantitative analysis to evaluate the site-specific diagnostic accuracy of FOBT not only for CRC, but 
(A) for detecting colorectal cancer using gFOBT

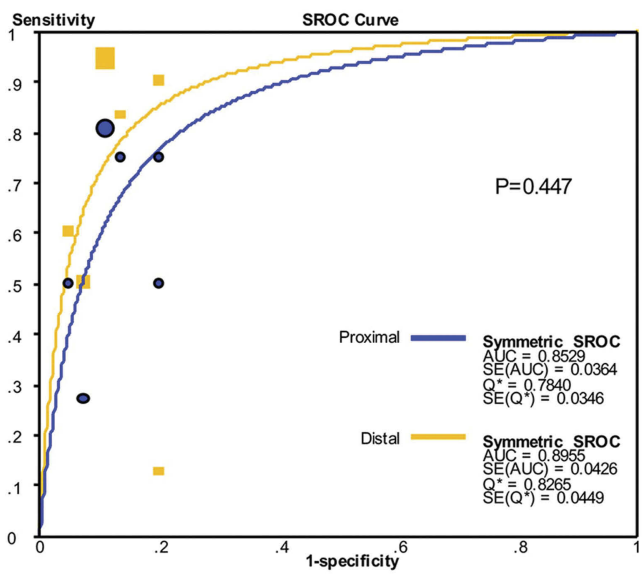

(C) for detecting advanced adenoma using gFOBT

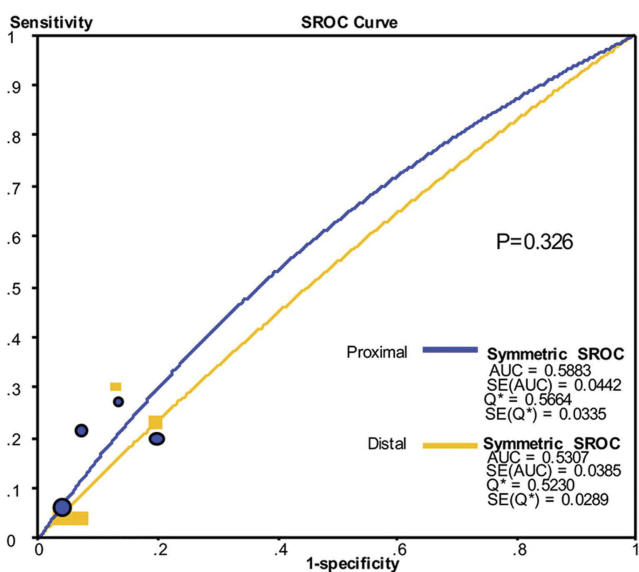

(E) for detecting advanced neoplasia using gFOBT

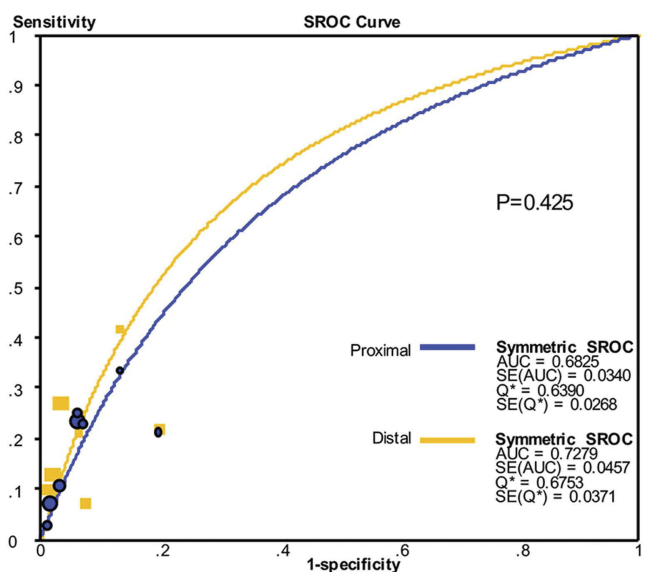

(B) for detecting colorectal cancer using iFOBT

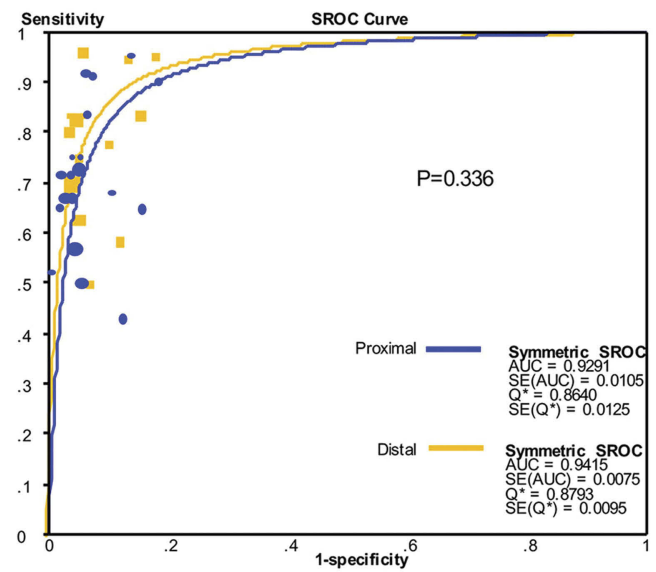

(D) for detecting advanced adenoma using iFOBT

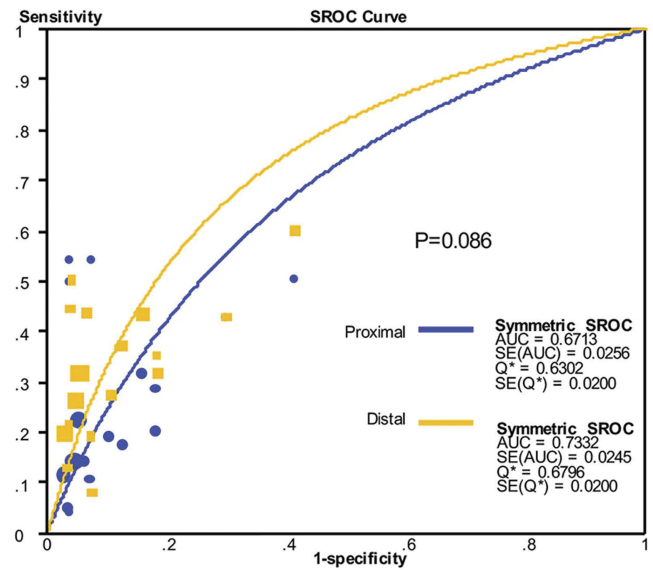

(F) for detecting advanced neoplasia using iFOBT

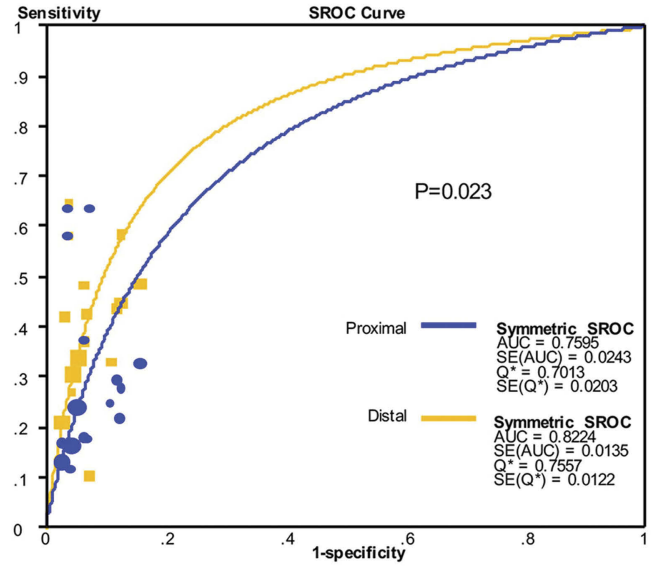

Figure 4 Comparison of SROCs for detecting colorectal neoplasms located in the proximal and distal colon/rectum, (A) for detecting colorectal cancer using guaiac-fecal occult blood test; (B) for detecting colorectal cancer using immunochemical fecal occult blood test; (C) for detecting advanced adenoma using guaiac-fecal occult blood test; (D) for detecting advanced adenoma using immunochemical fecal occult blood test; (E) for detecting advanced neoplasia using guaiac-fecal occult blood test; (F) for detecting advanced neoplasia using immunochemical fecal occult blood test.

Abbreviations: gFOBT, guaiac-fecal occult blood testing; iFOBT, immunochemical fecal occult blood testing. 
also for advanced adenomas. In addition, we performed subgroup analyses to assess the consistency of the results.

Our findings showed that both gFOBT and iFOBT had comparable sensitivities for detecting CRC located in the proximal or distal colorectum, indicating the overall good performance of FOBT in CRC screening. However, for advanced adenoma, iFOBT showed inferior sensitivity for detecting lesions located in the proximal colon than that for lesions in the distal colon/rectum. Advanced adenoma is the most important precursor of CRC. In populationbased CRC screening programs, timely detection of advanced adenoma and adoption of appropriate clinical intervention would strongly improve the overall survival of patients and even reduce the likelihood to develop CRC. ${ }^{46,47}$ Therefore, the relative lower sensitivity of iFOBT for detecting proximal advanced adenoma might affect the detection rate of advanced adenoma and limit its overall effectiveness in population-based CRC screening programs. Such an issue should be investigated and resolved in further studies.

To address the lower sensitivity for detecting colorectal neoplasms located in the proximal colon, further measures to optimize the scheme of FOBT-based CRC screening need to be explored. First, tests can be repeatedly performed. Our results were focused on a one-time application of iFOBT. Nevertheless, in a real-world setting, iFOBT-based screening programs are implemented with serial application of iFOBT over time (ie, every $1-2$ years), ${ }^{48,49}$ and a better detection of colorectal neoplasms is expected to be achieved in such a setting. ${ }^{49,50}$ Second, lower cut-off values for quantitative iFOBT could be applied. Previous studies have suggested that the sensitivity of iFOBT for advanced adenomas could be improved when the positivity threshold was lowered. It was shown that quantitative iFOBT, if using a low cut-off value less than $20 \mu \mathrm{g} \mathrm{hb} / \mathrm{g}$ feces, may offer improved sensitivity for the detection of CRC compared with a cut-off range from 20 to $50 \mu \mathrm{g} \mathrm{hb} / \mathrm{g}$ feces. ${ }^{9}$ Third, multiple fecal samples could be used for testing. Bleeding in advanced neoplasia cases may be missed with singlestool sampling due to the characteristic of intermittent bleeding. Therefore, multiple separate samples collected on several consecutive days could probably increase the sensitivity of the test. ${ }^{4,6}$ A study from the Netherlands found that 2-sample iFOBT screening using at least 1 positive test as a cut-off was associated with a higher detection rate for advanced neoplasia compared to 1 -sample iFOBT screening. ${ }^{51}$ However, concerns may be raised regarding the compliance, colonoscopy capacity, and over-screening after implementation of this screening scheme wherein improvements are made based on the mentioned suggestions. Hence, more studies on cost-effectiveness need to be performed to explore the balance between expense, medical resources, and yield of screening.

There are several strengths and limitations that need to be taken into consideration when interpreting our results. Strengths of our study include the adoption of rigorous inclusion and exclusion criteria in four widely used medical databases to ensure that all potential studies were included in this review. In addition, we compared the site-specific diagnostic accuracy of FOBT in detecting multiple outcomes, including CRC and advanced adenoma, which has not been done in previous review articles. Limitations of our studies include modest heterogeneity among the studies under review, due to different study designs, different study populations, and numerous FOBTs with different cut-off values. Therefore, we conducted subgroup analyses, which showed overall consistency with our main results that indicated to be reliable enough not to be influenced by the heterogeneity. Second, the sensitivity for detecting advanced neoplasms may be under- or overestimated because such an indicator is strongly affected by the proportion of CRC and advanced adenoma cases in the study population. Third, participants younger than 40 years old were enrolled into some studies, which may introduce spectrum bias into our analysis. Fourth, given the limited data on advanced adenoma, its scope was unrestricted and extended to include any colorectal adenoma in 2 studies. $^{28,32}$

Altogether, our study showed that iFOBT had higher sensitivity for detecting advanced colorectal neoplasms, especially advanced adenomas, located in the distal colon/rectum than that for those in the proximal colon. Further efforts should be made to develop customized schemes of CRC screening according to local program needs with enhanced detection of precursors to CRC in the proximal colon and enhanced potential of proximal CRC prevention, while maintaining or even increasing the cost-effectiveness yielded by present screening strategies.

\section{Author Contributions}

All authors contributed to data analysis, drafting and revising the article, gave final approval of the version to be published, and agree to be accountable for all aspects of the work. 


\section{Funding}

The study was supported by the National Natural Science Foundation (81703309) and CAMS Innovation Fund for Medical Science (2017-I2M-1-006). The sponsor had no role in the study design, in the collection, analysis, and interpretation of data.

\section{Disclosure}

The authors report no conflicts of interest in this work.

\section{References}

1. Bray F, Ferlay J, Soerjomataram I, Siegel RL, Torre LA, Jemal A. Global cancer statistics 2018: GLOBOCAN estimates of incidence and mortality worldwide for 36 cancers in 185 countries. CA Cancer J Clin. 2018;68(6):394-424. doi:10.3322/caac.21492

2. Elmunzer BJ, Hayward RA, Schoenfeld PS, Saini SD, Deshpande A, Waljee AK. Effect of flexible sigmoidoscopy-based screening on incidence and mortality of colorectal cancer: a systematic review and meta-analysis of randomized controlled trials. PLoS Med 2012;9(12):e1001352. doi:10.1371/journal.pmed.1001352

3. Shaukat A, Mongin SJ, Geisser MS, et al. Long-term mortality after screening for colorectal cancer. $N$ Engl J Med. 2013;369(12):1106-1114.

4. Robertson DJ, Lee JK, Boland CR, et al. Recommendations on fecal immunochemical testing to screen for colorectal neoplasia: a consensus statement by the US multi-society task force on colorectal cancer Gastroenterology. 2017;152(5):1217-1237 e1213. doi:10.1053/j. gastro.2016.08.053

5. Schreuders EH, Ruco A, Rabeneck L, et al. Colorectal cancer screening: a global overview of existing programmes. Gut. 2015;64 (10):1637-1649. doi:10.1136/gutjnl-2014-309086

6. Tinmouth J, Lansdorp-Vogelaar I, Allison JE. Faecal immunochemical tests versus guaiac faecal occult blood tests: what clinicians and colorectal cancer screening programme organisers need to know. Gut. 2015;64(8):1327-1337. doi:10.1136/gutjnl-2014-308074

7. Faivre J, Dancourt V, Lejeune C, et al. Reduction in colorectal cancer mortality by fecal occult blood screening in a French controlled study. Gastroenterology. 2004;126(7):1674-1680. doi:10.1053/j. gastro.2004.02.018

8. Zorzi M, Hassan C, Capodaglio G, et al. Long-term performance of colorectal cancer screening programmes based on the faecal immunochemical test. Gut. 2017;67(12):2124-2130. doi:10.1136/gutjnl2017-314753

9. Lee JK, Liles EG, Bent S, Levin TR, Corley DA. Accuracy of fecal immunochemical tests for colorectal cancer: systematic review and metaanalysis. Ann Intern Med. 2014;160(3):171. doi:10.7326/M13-1484

10. Haug U, Knudsen AB, Brenner H, Kuntz KM. Is fecal occult blood testing more sensitive for left- versus right-sided colorectal neoplasia? A systematic literature review. Expert Rev Mol Diagn. 2011;11 (6):605-616. doi:10.1586/erm.11.41

11. Hirai HW, Tsoi KK, Chan JY, et al. Systematic review with metaanalysis: faecal occult blood tests show lower colorectal cancer detection rates in the proximal colon in colonoscopy-verified diagnostic studies. Aliment Pharmacol Ther. 2016;43(7):755-764. doi:10.1111/apt.13556

12. Brenner H, Stock C, Hoffmeister M. Effect of screening sigmoidoscopy and screening colonoscopy on colorectal cancer incidence and mortality: systematic review and meta-analysis of randomised controlled trials and observational studies. BMJ. 2014;348:g2467. doi:10.1136/bmj.g2467
13. Moher DSL, Clarke M, Ghersi D, et al. Preferred reporting items for systematic review and meta-analysis protocols (PRISMA-P) 2015 statement. Syst Rev. 2015;4:1. doi:10.1186/2046-4053-4-1

14. Whiting PF, Rutjes AW, Westwood ME, et al. QUADAS-2: a revised tool for the quality assessment of diagnostic accuracy studies. Ann Intern Med. 2011;155(8):529-536. doi:10.7326/0003-4819-155-8201110180-00009

15. Thomas WM, Hardcastle JD, Jackson J, Pye G. Chemical and immunological testing for faecel occult blood: a comparison of two tests in symptomatic patients. Br J Cancer. 1992;65(4):618-620. doi:10.1038/ bjc. 1992.125

16. Hope RL, Chu G, Hope AH, Newcombe RG, Gillespie PE, Williams SJ. Comparison of three faecal occult blood tests in the detection of colorectal neoplasia. Gut. 1996;39(5):722-725. doi:10.1136/gut.39.5.722

17. Greenberg PD, Bertario L, Gnauck R, et al. A prospective multicenter evaluation of new fecal occult blood tests in patients undergoing colonoscopy. Am J Gastroenterol. 2000;95(5):1331-1338. doi:10.1111/j.15720241.2000.02032.x

18. Lieberman DA, Weiss DG. One-time screening for colorectal cancer with combined fecal occult-blood testing and examination of the distal colon. $N$ Engl J Med. 2001;345(8):555-560. doi:10.1056/NEJMoa010328

19. Nakama H, Zhang B, Fattah ASMA, Kamijo N, Zhang X. Characteristics of colorectal cancer that produce positive immunochemical occult blood test results on stool obtained by digital rectal examination. Can J Gastroenterol. 2001;15(4):227-230. doi:10.1155/2001/468125

20. Sung JJY, Chan FKL, Leung WK, et al. Screening for colorectal cancer in Chinese: comparison of fecal occult blood test, flexible sigmoidoscopy, and colonoscopy. Gastroenterology. 2003;124 (3):608-614. doi:10.1053/gast.2003.50090

21. Young GP, St John DJ, Cole SR, et al. Prescreening evaluation of a brush-based faecal immunochemical test for haemoglobin. $J$ Med Screen. 2003;10(3):123-128. doi:10.1177/096914130301000305

22. Morikawa T, Kato J, Yamaji Y, Wada R, Mitsushima T, Shiratori Y. A comparison of the immunochemical fecal occult blood test and total colonoscopy in the asymptomatic population. Gastroenterology. 2005;129(2):422-428. doi:10.1016/j.gastro.2005.05.056

23. Nakazato M, Yamano HO, Matsushita HO, et al. Immunologic fecal occult blood test for colorectal cancer screening. Japan Med Assoc J. 2006;49(5-6):203-207.

24. Ahlquist DA, Sargent DJ, Loprinzi CL, et al. Stool DNA and occult blood testing for screen detection of colorectal neoplasia. Ann Intern Med. 2008;149(7):441-450. doi:10.7326/0003-4819-149-7-200810070-00004

25. Shastri Y, Loitsch S, Hoepffner N, et al. Comparison of an established simple office-based immunological FOBT with fecal tumor pyruvate kinase type M2 (M2-PK) for colorectal cancer screening: prospective multicenter study. Am $J$ Gastroenterol. 2008;103 (6):1496-1504. doi:10.1111/j.1572-0241.2008.01824.x

26. Bjerregaard NC, Tottrup A, Sorensen HT, Laurberg S. Detection of colorectal cancer in symptomatic outpatients without visible rectal bleeding: validity of the fecal occult blood test. Clin Epidemiol. 2009;1:119-124.

27. Graser A, Stieber P, Nagel D, et al. Comparison of CT colonography, colonoscopy, sigmoidoscopy and faecal occult blood tests for the detection of advanced adenoma in an average risk population. Gut. 2009;58(2):241-248. doi:10.1136/gut.2008.156448

28. Hundt S, Haug U, Brenner H. Comparative evaluation of immunochemical fecal occult blood tests for colorectal adenoma detection. Ann Intern Med. 2009;150(3):162-169. doi:10.7326/0003-4819-150-3-200902030-00005

29. Haug U, Hundt S, Brenner H. Quantitative immunochemical fecal occult blood testing for colorectal adenoma detection: evaluation in the target population of screening and comparison with qualitative tests. $\mathrm{Am} \mathrm{J}$ Gastroenterol. 2010;105(3):682-690. doi:10.1038/ajg.2009.668

30. Oono Y, Iriguchi Y, Doi Y, et al. A retrospective study of immunochemical fecal occult blood testing for colorectal cancer detection. Clin Chim Acta. 2010;411(11-12):802-805. doi:10.1016/j. cca.2010.02.057 
31. Park DI, Ryu S, Kim YH, et al. Comparison of guaiac-based and quantitative immunochemical fecal occult blood testing in a population at average risk undergoing colorectal cancer screening. Am J Gastroenterol. 2010;105(9):2017-2025. doi:10.1038/ajg.2010.179

32. Haug U, Kuntz KM, Knudsen AB, Hundt S, Brenner H. Sensitivity of immunochemical faecal occult blood testing for detecting left-vs right-sided colorectal neoplasia. Br J Cancer. 2011;104(11):17791785. doi:10.1038/bjc. 2011.160

33. Khalid-de Bakker CAJ, Jonkers DMAE, Sanduleanu S, et al. Test performance of immunologic fecal occult blood testing and sigmoidoscopy compared with primary colonoscopy screening for colorectal advanced adenomas. Cancer Prev Res. 2011;4(10):1563-1571. doi:10.1158/1940-6207.CAPR-11-0076

34. de Wijkerslooth TR, Stoop EM, Bossuyt PM, et al. Immunochemical fecal occult blood testing is equally sensitive for proximal and distal advanced neoplasia. Am J Gastroenterol. 2012;107(10):1570-1578. doi:10.1038/ajg.2012.249

35. Wong CKW, Fedorak RN, Prosser CI, Stewart ME, Van Zanten SV, Sadowski DC. The sensitivity and specificity of guaiac and immunochemical fecal occult blood tests for the detection of advanced colonic adenomas and cancer. Int $J$ Colorectal Dis. 2012;27 (12):1657-1664. doi:10.1007/s00384-012-1518-3

36. Chiu HM, Lee YC, Tu CH, et al. Association between early stage colon neoplasms and false-negative results from the fecal immunochemical test. Clin Gastroenterol Hepatol. 2013;11(7):832-838e831832. doi:10.1016/j.cgh.2013.01.013

37. Kaul A, Shah A, Magill FH, Hawkins SA, Skaife P. Immunological faecal occult blood testing: A discriminatory test to identify colorectal cancer in symptomatic patients. Int J Surg. 2013;11(4):329-331. doi:10.1016/j.ijsu.2013.02.013

38. Koga Y, Yamazaki N, Yamamoto Y, et al. Fecal miR-106a is a useful marker for colorectal cancer patients with false-negative results in immunochemical fecal occult blood test. Cancer Epidemiol Biomarkers Prev. 2013;22(10):1844-1852. doi:10.1158/1055-9965.EPI-13-0512

39. Lee YC, Chiu HM, Chiang TH, et al. Accuracy of faecal occult blood test and Helicobacter pylori stool antigen test for detection of upper gastrointestinal lesions. BMJ Open. 2013;3(10):e003989. doi:10.1136/bmjopen2013-003989

40. Kim BC, Joo J, Chang HJ, et al. A predictive model combining fecal calgranulin $\mathrm{B}$ and fecal occult blood tests can improve the diagnosis of colorectal cancer. PLoS One. 2014;9(9):e106182. doi:10.1371/ journal.pone. 0106182
41. Imperiale TF, Ransohoff DF, Itzkowitz SH, et al. Multitarget stool DNA testing for colorectal-cancer screening. $N$ Engl J Med. 2014;370(14):1287-1297. doi:10.1056/NEJMoa1311194

42. Castro I, Estevez P, Cubiella J, et al. Diagnostic performance of fecal immunochemical test and sigmoidoscopy for advanced right-sided colorectal neoplasms. Dig Dis Sci. 2015;60(5):1424-1432. doi:10.1007/s10620-014-3434-6

43. Kim NH, Yang HJ, Park SK, et al. Does low threshold value use improve proximal neoplasia detection by fecal immunochemical test? Dig Dis Sci. 2016;61(9):2685-2693. doi:10.1007/s10620-0164169-3

44. Brenner H, Niedermaier T, Chen H. Strong subsite-specific variation in detecting advanced adenomas by fecal immunochemical testing for hemoglobin. Int $J$ Cancer. 2017;140(9):2015-2022. doi:10.1002/ ijc.30629

45. Jung YS, Park CH, Kim NH, Park JH, Park DI, Sohn CI. Clinical risk stratification model for advanced colorectal neoplasia in persons with negative fecal immunochemical test results. PLoS One. 2018;13(1): e0191125. doi:10.1371/journal.pone.0191125

46. Zauber AG, Winawer SJ, O’Brien MJ, et al. Colonoscopic polypectomy and long-term prevention of colorectal-cancer deaths. $N$ Engl J Med. 2012;366(8):687-696. doi:10.1056/NEJMoa1100370

47. Click B, Pinsky PF, Hickey T, Doroudi M, Schoen RE. Association of colonoscopy adenoma findings with long-term colorectal cancer incidence. JAMA. 2018;319(19):2021-2031. doi:10.1001/jama.2018.5809

48. Force USPST, Bibbins-Domingo K, Grossman DC, et al. Screening for colorectal cancer: US preventive services task force recommendation statement. JAMA. 2016;315(23):2564-2575. doi:10.1001/ jama.2016.5989

49. Logan RF, Patnick J, Nickerson C, Coleman L, Rutter MD, von Wagner C. Outcomes of the Bowel Cancer Screening Programme (BCSP) in England after the first 1 million tests. Gut. 2012;61 (10):1439-1446. doi:10.1136/gutjnl-2011-300843

50. Crotta S, Segnan N, Paganin S, Dagnes B, Rosset R, Senore C. High rate of advanced adenoma detection in 4 rounds of colorectal cancer screening with the fecal immunochemical test. Clin Gastroenterol Hepatol. 2012;10(6):633-638. doi:10.1016/j.cgh. 2012.02.030

51. van Roon AH, Wilschut JA, Hol L, et al. Diagnostic yield improves with collection of 2 samples in fecal immunochemical test screening without affecting attendance. Clin Gastroenterol Hepatol. 2011;9 (4):333-339. doi:10.1016/j.cgh.2010.12.012
Clinical Epidemiology

\section{Publish your work in this journal}

Clinical Epidemiology is an international, peer-reviewed, open access, online journal focusing on disease and drug epidemiology, identification of risk factors and screening procedures to develop optimal preventative initiatives and programs. Specific topics include: diagnosis, prognosis, treatment, screening, prevention, risk factor modification, systematic reviews, risk \& safety of medical interventions, epidemiology \& biostatistical methods, and evaluation of guidelines, translational medicine, health policies \& economic evaluations. The manuscript management system is completely online and includes a very quick and fair peer-review system, which is all easy to use. 